\title{
Degree of polarization and quantum-mechanical purity
}

\author{
H. Moya-Cessa \\ hmmc@inaoep.mx
}

\section{J.R. Moya-Cessa}

\section{J. E. A. Landgrave}

\section{G. Martínez-Niconoff}

\section{A. Pérez-Leija}

\section{A. T. Friberg}

\author{
INAOE, Coordinación de Optica, Apdo. Postal 51 y 216, 72000 Puebla, Pue., Mexico \\ Centro de Investigaciones en Optica, A.C., Apartado Postal 1-948, 37000 León, Gto., Mexico
}

Centro de Investigaciones en Optica, A.C., Apartado Postal 1-948, 37000 León, Gto., Mexico

INAOE, Coordinación de Optica, Apdo. Postal 51 y 216, 72000 Puebla, Pue., Mexico

INAOE, Coordinación de Optica, Apdo. Postal 51 y 216, 72000 Puebla, Pue., Mexico
Department of Engineering Physics Helsinki University of Technology (TKK), P.O. Box 3500, FI-02015 TKK, Finland

Department of Physics and Mathematics, University of Joensuu, P.O. Box 111, FI-80101 Joensuu, Finland

Department of Microelectronics and Applied Physics, Royal Institute of Technology (KTH), Electrum 229, SE-164 40 Kista, Sweden

The purity parameter is used in quantum mechanics to discriminate pure states from mixed states. We employ this concept to define a degree of polarization for general, three-dimensional, classical random electric fields. Our approach leads to a result that is identical with a recent definition obtained by a decomposition of the polarization matrix in terms of the Gell-Mann matrices. We also give an expression for this degree of polarization based on the constituent two-dimensional subsystems. [D0I: 10.2971/je0s.2008.08014]

Keywords: Polarization, entropy, purity

\section{INTRODUCTION}

The fact that the paraxial approximation to the Helmholtz equation leads to a Schrödinger equation may be responsible for the many analogies that are found between quantum physics and classical optics. Along these lines there are several works producing such comparisons: Crasser et al. [1] have asked if Fresnel optics is quantum mechanics in phase space and Man'ko et al. [2] have shown how to realize quantum gates in optical fibers, i.e., by using quantumlike systems. The quantumlike bits (qulbits) are associated with light modes in the fiber and quantum gates with segments of the fiber providing unitary transformations of the mode structure. More recently, Chávez-Cerda et al. [3] have applied quantumoptical methods to solve propagation problems that recreate quantum-mechanical systems. Following these ideas, here we will propose an analogy between polarization in classical statistical optics and purity in quantum mechanics.

Characterization of the correlations that exist between the orthogonal components of an electromagnetic field is commonly obtained via the degree of polarization. The polarization state of a fluctuating electric field is traditionally described in terms of the $2 \times 2$ coherency (more appropriately, polarization) matrix or by using the related four Stokes parameters. [4]-[6] However, only electric fields with nearly planar wave fronts, such as well-collimated, uniform optical beams or radiated wide-angle far fields, can assume such a two-dimensional (2D) formalism. Those waves can be represented by two orthogonal electric-field components, but an arbitrary field is generally composed of three components. Common examples of such more general wave fields are electromagnetic near fields and high-numerical-aperture focused waves. The polarization state of an arbitrary random electromagnetic field, at a point, is fully characterized by the $3 \times 3$ polarization matrix formed by the three electric-field components $E_{i}, i=(x, y, z)$. Recently, based on a decomposition of the polarization matrix in terms of the Gell-Mann matrices, [7] Setälä et al. [8] have proposed a definition for the degree of polarization (3D) that reads

$$
P_{3}^{2}=\frac{3}{2}\left[\frac{\operatorname{tr}\left(\Phi_{3}^{2}\right)}{\operatorname{tr}^{2}\left(\Phi_{3}\right)}-\frac{1}{3}\right],
$$

where $\Phi_{3}$ is the 3D polarization matrix whose elements are

$$
\phi_{i j}(\mathbf{r}, \omega)=\left\langle E_{i}^{*}(\mathbf{r}, \omega) E_{j}(\mathbf{r}, \omega)\right\rangle,
$$

with $(i, j)=(x, y, z)$ and the angle brackets denoting ensemble average. Similar expressions have earlier been presented by Barakat [9] and Samson, [10] though without explicit association with a general electric field's polarization state. The 
notion of the degree of polarization has subsequently been extensively studied in the context of arbitrary fluctuating fields; for recent reviews see, e.g., [11] and [12]. The representation of the $3 \times 3$ polarization matrix by the Gell-Mann matrices is formally analogous to the expansion of the $2 \times 2$ polarization matrix by the Pauli matrices, [8] leading to the Stokes parameters and the usual degree of polarization for beams. [4]-[6] The expression in Eq. (1) has also been obtained by Luis [13] by formulating the degree of polarization as a vector-space distance between the field's polarization matrix and the identity matrix (that represents completely unpolarized light).

In this contribution we show that on borrowing the concept of purity from quantum mechanics, one directly obtains the degree of polarization given in Eq. (1).

\section{ENTROPY AND PURITY}

In quantum physics, a system is said to be in a pure state $|\psi\rangle$ if the associated density matrix, $\hat{\rho}$, is of the form

$$
\hat{\rho}^{(p)}=|\psi\rangle\langle\psi| ;
$$

otherwise,

$$
\hat{\rho}^{(m)}=\sum_{\alpha} p_{\alpha}\left|\psi_{\alpha}\right\rangle\left\langle\psi_{\alpha}\right|
$$

where $p_{\alpha}$ is the probability of state $\left|\psi_{\alpha}\right\rangle$, and the system is in a mixed state $\left(\sum_{\alpha} p_{\alpha}=1, p_{\alpha} \geq 0\right)$. The system's state of purity can be assessed either by calculating the entropy

$$
S=-\operatorname{tr}\{\hat{\rho} \ln \hat{\rho}\},
$$

or by evaluating the purity parameter

$$
\xi=1-\operatorname{tr}\left\{\hat{\rho}^{2}\right\}
$$

The purity parameter discriminates uniquely between mixed and pure states and is a lower bound for the entropy, $\xi \leq S$. $[14,15]$ If the system is in a pure state, one has $\hat{\rho}_{p}^{2}=\hat{\rho}_{p}$ (in fact, $\hat{\rho}_{p}$ is idempotent [16]). By normalization the trace of the density matrix is unity, $\operatorname{tr}\{\hat{\rho}\}=1$, giving a purity parameter for a pure state equal to zero, i.e., $\xi_{p}=0$. The polarization matrix shares many of the properties of the density matrix; $[16,17]$ in particular, they are Hermitian and non-negative definite. For fully polarized light the normalized (2D or 3D) polarization matrix assumes the form [18] $\Lambda_{p}=\hat{\mathbf{e}} \hat{\mathbf{e}}^{\dagger}$, where $\hat{\mathbf{e}}=\mathbf{E} /|\mathbf{E}|$ and $\mathbf{E}$ is the (column vector) electric field. It then follows at once that for light in a fully polarized state $\Lambda_{p}^{2}=\Lambda_{p}$, in analogy with the density matrix of a pure state. We make use of the mathematical similarity between the density and polarization matrices and convert the purity parameter of quantum mechanics to a degree of polarization for arbitrary random electromagnetic fields.

We note that while pure states and fully polarized fields are analogous, quantum mechanics and classical optics have in a sense opposite definitions; the degree of polarization equal to one means a completely polarized field but the degree of purity equal to zero corresponds to a quantum-mechanically pure state. Thus, the quantity in Eq. (6) is in reality a measure of the 'impurity' of the system. In order to produce a customary degree of polarization, we first have to rewrite the purity parameter $\xi$ in such a way that for a maximally mixed state the parameter is zero, while for a pure state it is one. We do this generally in Appendix A and the result, $\xi_{N}$, is given by Eq. (A.4). In what follows, we apply this result to the cases of random $2 \mathrm{D}$ and $3 \mathrm{D}$ electromagnetic fields.

\section{2D AND 3D DEGREES OF POLARIZATION}

2D fields: For beam-like, fluctuating classical electric waves the degree of polarization, from Eq. (A.4), then is

$$
\xi_{\Phi}^{(2)}=\sqrt{1-2\left(1-\operatorname{tr}\left\{\Lambda_{2}^{2}\right\}\right)}
$$

where the matrix $\Lambda_{2}=\Phi_{2} / \operatorname{tr}\left\{\Phi_{2}\right\}$ and $\Phi_{2}$ is given by the elements in Eq. (2). Thus $\Lambda_{2}$ is trace-normalized, just as the density matrix in quantum mechanics. The factor 2 in Eq. (7) is explained in Appendix A. In order to show that this definition coincides with the usual expression for the $2 \mathrm{D}$ degree of polarization, we use the Cayley-Hamilton theorem [19]. It states that every square matrix obeys its eigenvalue equation, i.e.,

$$
\Lambda_{2}^{2}-\Lambda_{2}+\frac{\operatorname{det}\left\{\Phi_{2}\right\}}{\operatorname{tr}^{2}\left\{\Phi_{2}\right\}} I_{2}=0
$$

with $I_{2}$ being the $2 \mathrm{D}$ identity matrix. From Eq. (8) we readily find $\operatorname{tr}\left\{\Lambda_{2}^{2}\right\}$ and subsequently we can calculate the degree of polarization in the 2D case by using Eq. (7), obtaining

$$
\xi_{\Phi}^{(2)}=\sqrt{1-\frac{4 \operatorname{det}\left\{\Phi_{2}\right\}}{\operatorname{tr}^{2}\left\{\Phi_{2}\right\}}}
$$

in agreement with the conventional result. [4]-[6]

$3 D$ fields: For general 3D random electric fields the degree of polarization is defined, on the basis of Eq. (A.4), as

$$
\xi_{\Phi}^{(3)}=\sqrt{1-\frac{3}{2}\left(1-\operatorname{tr}\left\{\Lambda_{3}^{2}\right\}\right)}
$$

where $\Lambda_{3}=\Phi_{3} / \operatorname{tr}\left\{\Phi_{3}\right\}$ and, again, the factor $3 / 2$ is explained in Appendix A. It can readily be seen that Eq. (10) is nothing but the degree of polarization given in Eq. (1). Further, by observing that

$$
\operatorname{tr}\left\{\Lambda_{3}^{2}\right\}=\lambda_{11}^{2}+\lambda_{22}^{2}+\lambda_{33}^{2}+2 \sum_{i<j}\left|\lambda_{i j}\right|^{2}
$$

where $\lambda_{i j}$ are the elements of the matrix $\Lambda_{3}$, and noting that $\lambda_{11}+\lambda_{22}+\lambda_{33}=1$, we may re-write Eq. (10) as an expression similar to Eq. (9), viz.,

$$
\xi_{\Phi}^{(3)}=\sqrt{1-3 \frac{\sum_{i<j}\left|\begin{array}{cc}
\phi_{i i} & \phi_{i j} \\
\phi_{j i} & \phi_{j j}
\end{array}\right|}{\operatorname{tr}^{2}\left\{\Phi_{3}\right\}}} .
$$

This equation clearly shows that an important ingredient for the degree of polarization for 3D fields are the different 2D subsystems that can be formed from the 3D case. 


\section{CONCLUSIONS}

In summary, by using the concept of purity in quantum mechanics, we have introduced a degree of polarization when the full $3 \times 3$ (spectral) polarization matrix for classical electric fields is considered. This degree of polarization coincides with the definition given earlier in a different way by Setälä et al. [8] Our analysis brings into evidence the close analogy between the quantum-mechanical purity and the polarimetric purity in classical electromagnetic fields characterized by the degree of polarization.

\section{ACKNOWLEDGEMENTS}

The authors from Mexico thank partial support from CONACYT. ATF acknowledges funding from the Academy of Finland (under Finland Distinguished Professorship program) and the Swedish Foundation for Strategic Research (SSF).

\section{A $n$-LEVEL SYSTEM}

For the sake of completeness, we show briefly how to obtain a properly normalized purity parameter for an $n$-level quantum-mechanical system, i.e., a parameter that takes on a value zero for a maximally mixed state and one for a pure state. The density matrix for an $n$-level system is generally given by (see also Ref. [16])

$$
\hat{\rho}_{n}=\left(\begin{array}{cccc}
\rho_{11} & \rho_{12} & \ldots & \rho_{1 n} \\
\rho_{21} & \rho_{22} & \ldots & \rho_{2 n} \\
\ldots & \ldots & \ldots & \ldots \\
\rho_{n 1} & \rho_{n 2} & \ldots & \rho_{n n}
\end{array}\right) .
$$

A completely mixed state is given by the matrix

$$
\hat{\rho}_{n}=\left(\begin{array}{llll}
\frac{1}{n} & 0 & \ldots & 0 \\
0 & \frac{1}{n} & \ldots & 0 \\
\ldots & \ldots & \ldots & \ldots \\
0 & 0 & \ldots & \frac{1}{n}
\end{array}\right)
$$

such that its square is

$$
\hat{\rho}_{n}^{2}=\left(\begin{array}{cccc}
\frac{1}{n^{2}} & 0 & \ldots & 0 \\
0 & \frac{1}{n^{2}} & \ldots & 0 \\
\ldots & \ldots & \ldots & \ldots \\
0 & 0 & \ldots & \frac{1}{n^{2}}
\end{array}\right) .
$$

The purity parameter defined in Eq. (6) is readily calculated as $\xi_{n}=1-\operatorname{tr}\left\{\hat{\rho}_{n}^{2}\right\}=1-1 / n$. To normalize $\xi$ first so that it gives one for a maximally mixed state, we have to multiply it by $n /(n-1)$. This explains the factor 2 in the $2 \mathrm{D}$ case and $3 / 2$ in the $3 \mathrm{D}$ case. To invert the direction, we subtract this normalized parameter from unity. Thus we have an expression that has the right limiting values and is bounded between zero and one, i.e.,

$$
\xi_{\mathrm{N}}^{2}=1-\frac{n}{n-1}\left(1-\operatorname{tr}\left\{\hat{\rho}^{2}\right\}\right) .
$$

The power of $\xi_{\mathrm{N}}$ on the left-hand side could, in principle, be arbitrary but only the square yields the correct, well-known result for the degree of polarization of beamlike fields, as was demonstrated in the 2D case above.
Consider now the following density matrix for a 3-level system:

$$
\hat{\rho}_{3}=\left(\begin{array}{lll}
A & 0 & 0 \\
0 & A & 0 \\
0 & 0 & B
\end{array}\right),
$$

with $2 A+B=1$ and $A \neq B$. It corresponds to a (nonmaximally) mixed state. When $B$ tends to zero, the system seems to reduce to a (completely mixed) two-level system; however, this is not the case as the system still is a $3 \mathrm{D}$ one, with information about it: the 3rd level (represented by element $\rho_{33}$ ) is not occupied (at any time).

Finally, it is worth mentioning that for an $n \times n$ polarization matrix $\Phi_{n}$, as in Barakat's case, [16] the 'degree of polarization' from Eq. (A.4) takes on the form

$$
\xi_{\Phi}^{(n)}=\sqrt{1-\frac{n}{n-1} \frac{2 \sum_{i<j}\left|\begin{array}{ll}
\phi_{i i} & \phi_{i j} \\
\phi_{j i} & \phi_{j j}
\end{array}\right|}{\operatorname{tr}^{2}\left\{\Phi_{n}\right\}}} .
$$

\section{References}

[1] 0. Crasser, H. Mack, and W.P. Schleich, "Could Fresnel optics be quantum mechanics in phase space?" Fluct. Noise Lett. 4, L43-L51 (2004).

[2] M. A. Man'ko, V. I. Man'ko, and R. Vilela Mendes, "Quantum computation by quantumlike systems" Phys. Lett. A 288, 132-138 (2001).

[3] S. Chávez-Cerda, J.R. Moya-Cessa, and H. Moya-Cessa, “Quantumlike systems in classical optics: applications of quantum optical methods" J. Opt. Soc. Am. B 24, 404-407 (2007).

[4] C. Brosseau, Fundamentals of polarized light: A statistical optics approach (Wiley, New York, 1998).

[5] L. Mandel and E. Wolf, Optical coherence and quantum optics (Cambridge University Press, Cambridge, UK, 1995).

[6] M. Born and E. Wolf, Principles of optics, 7th ed. (Cambridge University Press, Cambridge, UK, 1999).

[7] M. Gell-Mann and Y. Ne'eman, The eightfold way (Benjamin, New York, 1964).

[8] T. Setälä, A. Shevchenko, M. Kaivola, and A.T. Friberg, "Degree of polarization for optical near fields" Phys. Rev. E 66, 016615 (2002).

[9] R. Barakat, "Degree of polarization and the principal idempotents of the coherency matrix" Opt. Commun. 23, 147-150 (1977).

[10] J. C. Samson and J. V. Olson, "Some comments on the descriptions of the polarization states of waves" Geophys. J. Roy. Astr. S. 61, 115 Ü129 (1980).

[11] C. Brosseau and A. Dogariu, "Symmetry properties and polarization descriptors for an arbitrary electromagnetic wavefield" in Progress in Optics, E. Wolf, ed., Vol. 49, p. 315 (Elsevier, Amsterdam, 2006).

[12] J.J. Cil, "Polarimetric characterization of light and media - Physical quantities involved in polarimetric phenomena" Eur. Phys. J. Appl. Phys. 40, 1-47 (2007).

[13] A. Luis, "Degree of polarization for three-dimensional fields as a distance between correlation matrices" 0pt. Commun. 253, 10-14 (2005). 
[14] U. Leonhardt, Measuring the quantum state of light (Cambridge University Press, Cambridge, UK, 1997).

[15] H. Moya-Cessa, "Decoherence in atom-field interactions: A treatment using superoperator techniques" Phys. Rep. 432, 1-41 (2006).

[16] R. Barakat, "n-fold polarization measures and associated thermodynamic entropy of $\mathrm{N}$ partially coherent pencils of radiation" $0 \mathrm{pt}$. Acta 30, 1171-1182 (1983).
[17] U. Fano, "Description of states in quantum mechanics by density matrix and operator techniques" Rev. Mod. Phys. 29, 74-93 (1957).

[18] J. Ellis, A. Dogariu, S. Ponomarenko, and E. Wolf, "Correlation matrix of a completely polarized, statistically stationary electromagnetic field" Opt. Lett. 29, 1536-1538 (2004).

[19] R. B. J. T. Allenby, Linear algebra, in Modular Mathematics (Butterworth-Heinemann, 0xford, UK, 1995). 